\begin{tabular}{|l|l|l|l|l|l|}
\hline MUNIBE Antropologia-Arkeologia & $\mathrm{n}^{\circ} 65$ & $67-78$ & DONOSTIA & 2014 & ISSN 1132-2217 • eISSN 2172-4555 \\
\hline
\end{tabular}

\title{
Entre lapas: primera valoración de los restos de origen marino del yacimiento holoceno de $J 3$ (Hondarribia, País Vasco)
}

\author{
Among the limpets: initial assessment of marine remains \\ at the Holocene site of J3 (Hondarribia, Basque Country)
}

KEY WORDS: Exploitation of marine resources, Prehistory, Holocene, Mesolithic, Basque Country. PALABRAS CLAVES: Explotación de recursos marinos, Prehistoria, Holoceno, Mesolítico, País Vasco. GAKO-HITZAK: Itsasoko baliabideen ustiapena, Historiaurrea, Holozenoa, Mesolitoa, Euskal Herria.

\section{Esteban ÁLVAREZ-FERNÁNDEZ(1), María José IRIARTE-CHIAPUSSO(2,3), Álvaro ARRIZABALAGA(3), Inmaculada BARRERA-MELLADO(4), René-Pierre CARRIOL(5), María José FERNÁNDEZ-GÓMEZ(4)y Sonia GABRIEL(6)}

\section{ABSTRACT}

The site of J3 (Hondarribia, Guipuzkoa) is located in Sierra de Jaizkibel (Basque Country, Spain), only 200m from the shore. In this rockshelter, several archaeological levels dated in the early Holocene (Mesolithic) have been excavated. In Levels B, C, D and F, huge accumulations of archaeozoological remains were found, mainly the shells of marine molluscs, which means that the deposit is regarded as a "stratified shell-midden", one of the few examples known in Cantabrian Spain dated in this period. It is estimated that in the trial excavation carried out (ca. $\left.1.5 \mathrm{~m}^{2}\right)$, the number of remains could reach half a million.

This paper presents the first data about the use of marine resources at $\mathrm{J} 3$, based on a comprehensive study of the remains in a sample taken from the deposit (from a sector $33 \times 33 \mathrm{~cm}$ in size). The number of remains of molluscs, crustaceans, echinoderms and fish amounts to 45,200.

The archaeo-faunal composition is similar in the four levels. The most abundant remains are gastropod shells, which represent $>95 \%$ of the remains in the four levels. Limpets make up nearly $95 \%$ of the molluscs in each level, with $P$. vulgata, $P$. depressa, and to a lesser extent, $P$. ulyssiponensis. The remaining percentage consists of Phorcus lineatus, while other gastropods (Stramonita haemastoma, Gillbbula sp., etc.) are extremely scarce.

The remains of other marine animals have been recovered: plates of the goose barnacle Pollicipes pollicipes (only in Levels B, C and D), and balanomorphs (Chthamalus stellatus, Chthamalus montagui and Perforatus perforatus perforatus), spines and carapace fragments of the purple sea urchin Paracentrotus lividus, and fish bones (Sparidae)

Studies based on the epifauna and taphonomic analyses indicate that the limpets, top shells, barnacles and fish were gathered and caught as food on the shore near the rock-shelter, in the upper, middle and lower inter-tidal zone. The low tide zone may also have been visited. Continuity is seen in the exploitation of the marine environment throughout the sequence, in both Mesolithic and post-Mesolithic levels. These levels are interpreted as middens, where food waste was deposited. However, the remains of a buried human individual were documented in Level D.

The biometric study of the most abundant remains of molluscs and crustaceans (limpet shells and barnacle plates) does not reveal any evidence supporting the over-exploitation of the marine resources.

The use of advanced methodology, both in the excavation process at $\mathrm{J} 3$ and in the processing and study of the remains in the laboratory (based mainly in the taxonomical identification, quantification, taphonomic alterations and biometry), means that $\mathrm{J} 3$ is a benchmark site for understanding the role marine resources played in the diet of early Holocene human groups, not only in northern Spain but also in south-west Europe.

\section{RESUMEN}

Uno de los yacimientos prehistóricos más importantes documentados en la Sierra de Jaizkibel es J3. Situado a tan solo $200 \mathrm{~m}$ de la costa, en este abrigo se han excavado diferentes niveles arqueológicos datados a comienzos del Holoceno (Mesolítico). En los niveles B, C, D y F se documentaron enormes acumulaciones de conchas, lo que hace que el yacimiento sea considerado como un "conchero" estratificado. Calculamos que, en el sondeo realizado (ca. 1,5 m²), el número de restos podría alcanzar el medio millón. En este artículo presentamos los primeros datos sobre la explotación de recursos marinos procedentes de J3. Se ha estudiado una muestra del yacimiento. En los cuatro niveles la composición arqueofaunística es similar. Los restos más abundantes son las conchas de gasterópodos (casi exclusivamente lapas, Phorcus lineatus). También hay evidencias de crustáceos (placas de percebes y de balanos), de equinodermos (erizos) y de peces (espáridos). La presencia de estos restos arqueofaunísticos en J3 indica que los grupos humanos marisqueaban diferentes animales marinos en la zona intermareal cercana al yacimiento.

\footnotetext{
(1) Dep. Prehistoria, $\mathrm{H}^{a}$ Antigua y Arqueología, GIR PREHUSAL. Fac. de Geografía e Historia. Universidad de Salamanca. Cerrada de Serranos S/N, 37002 Salamanca. epanik@usal.es

(2) IKERBASQUE, Facultad de Letras (UPV/EHU), Tomás y Valiente s/n, 01006 Vitoria (España).

(3) Área de Prehistoria -Universidad del País Vasco, Facultad de Letras (UPV-EHU), Tomás y Valiente s/n, 01006 Vitoria (España).

(4) Dpto. Estadística. Facultad de Medicina. Universidad de Salamanca (campus Miguel de Unamuno). C. Alfonso X el Sabio S/N, 37007 Salamanca (España).

(5) Muséum National d’Histoire Naturelle, Département Histoire de la Terre, Case Postale 38, 57 rue Cuvier, F-75231 Paris Cedex 5, (Francia).

(6) Direcçao Geral do Património Cultural-(CDGPC-LARC) y EnvArch-CIBIO-INBIO. Rua da Bica do Marquês, 2. 1300-087 Lisbos (Portugal).
} 


\section{LABURPENA}

J3 Jaizkibel mendian orain arte ezagututako historiaurreko aztarnategi garrantzitsuenetako bat da. Itsasaldetik 200 metro eskasera dagoen babesleku honetan Holozenoko (Mesolitoa) hainbat maila arkeologiko induskatu dituzte. B, C, D eta F mailetan izugarrizko maskor-metaketak dokumentatu dituzte, eta horregatik, aztarnategia hainbat geruzatako "maskorleku" dela diote. Egindako zundaketaren arabera (1,5 m² inguru), aurkitutako aztarnak milioi erdira heltzen direla kalkulatu dugu. Artikulu honetan J3ko itsasoko baliabideen ustiapenaren lehendabiziko datuak aurkezten ditugu. Aztarnategiaren lagin bat aztertu dugu. Arkeofaunaren konposizioa antzekoa da lau mailetan. Gastropodoen maskorrenak dira ugarien dauden aztarnak (ia lapenak soilik, Phorcus lineatus). Bada, halaber, krustazeoen (lanperna eta balano plakak), ekinodermatuen (itsas trikuak) eta arrainen (esparidoak) aztarnarik. J3 aztarnategian halako arkeofauna aztarnak azaldu izanak adierazten du gizataldeak hainbat itsas animalia hartzen ibiltzen zirela aztarnategi ondoko marearteko tokietan.

\section{1.- INTRODUCCIÓN}

Los "concheros" son definidos como acumulaciones formadas, fundamentalmente, por conchas moluscos y de otros restos de animales marinos, que han sido consumidos en diferentes momentos de la ocupación de los yacimientos.

A pesar de que en la Región Cantábrica se han documentado más de un centenar de estas acumulaciones antrópicas, fundamentalmente en cuevas, poco son los que se han estudiado en profundidad. Este tipo de contextos se ubican a finales del Pleistoceno superior y comienzos del Holoceno, si bien es cierto, que los más abundantes se documentan en el Mesolítico, sobre todo, en el Este de Asturias (FANO, 2007).

Entre yacimientos holocenos que cuentan con abundantes restos de invertebrados hay que citar, por ejemplo, La Poza I'Egua (ARIAS et al., 2007) y Mazaculos II (GUTIÉRREZ, 2009) en Asturias, La Garma A (ÁLVAREZ-FERNÁNDEZ, 2013) y Los Gitanos (ÁLVAREZ-FERNÁNDEZ et al., 2014), en Cantabria y Santimamiñe (GUTIÉRREZ, 2009), en el País Vasco (Figura 1).

J3 es el único yacimiento de la provincia de Gipuzkoa que puede ser considerado como conchero. Aquí se han documentado cuatro niveles de acumulaciones de inverte- brados (niveles B, C, D y F), los dos más antiguos, datados en el Mesolítico, los dos más recientes adscritos a un momento posterior a este periodo (IRIARTE et al., 2005, 2010).

En este artículo se ofrecen los primeros datos sobre los recursos de origen marino procedentes de $\mathrm{J} 3$, relativos a la composición malacológica de cada uno de los niveles, indicando en que zona de la costa pudieron haber sido recogidos. También se ofrecen datos sobre la conservación de los restos y los primeros resultados de tipo biométrico de los moluscos.

\section{2.- MÉTODOS}

En J3 se abrió en los años 2002 y 2003 una superficie de excavación de unos 1,5 m². El cuadro K12 se excavó en su totalidad. Se dividió en 9 subcuadros de $33 \times 33 \mathrm{~cm}$. Se alcanzó una potencia máxima de 1,5 m y se documentaron los siguientes niveles arqueológicos (IRIARTE et al., 2010):

- Nivel A: Nivel superficial.

- Nivel B. Primer nivel de conchero, con una fecha de $10 \pm 70 \mathrm{BP}$

- Nivel C. Segundo nivel de conchero.

- Nivel D: Tercer nivel de conchero, cuyo techo está datado en $7770 \pm 50 \mathrm{BP}$. El enterramiento documentado en este nivel se dató en $8300 \pm 50 \mathrm{BP}$.

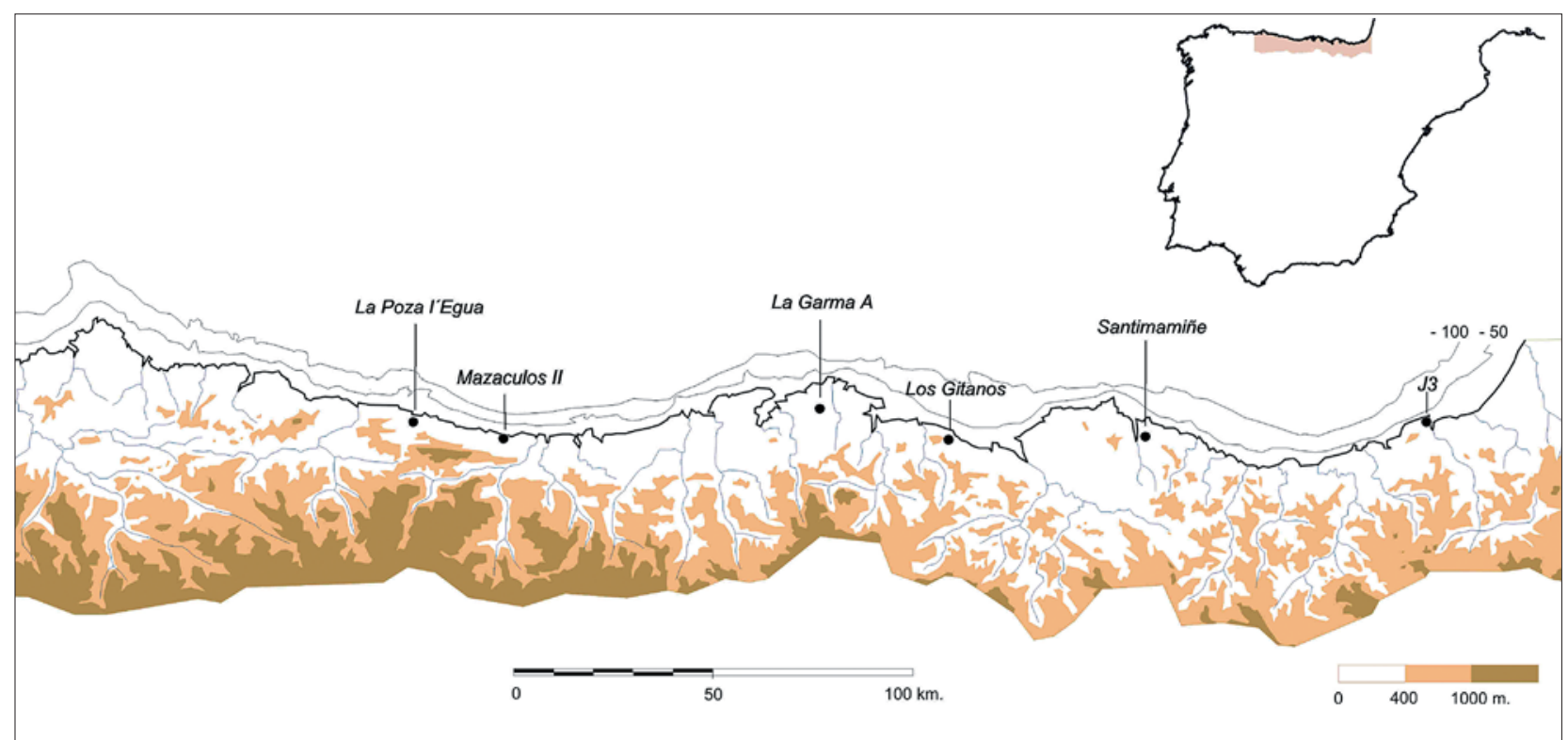

Fig. 1. Principales yacimientos holocenos de la región cantábrica con acumulaciones de conchas. / Main Holocene sites with shell-middens in northern Spain. 
- Nivel E: Nivel sin conchero

- Nivel F: Cuarto nivel de conchero, del que se dispone de dos fechas: $8190 \pm 100$ y $7790 \pm 130$ BP.

- Nivel G: Nivel sin conchero, por debajo del cual aparece la roca madre. Se obtuvieron dos fechas: $8470 \pm 100$ y $8470 \pm 50 \mathrm{BP}$

En el cuadro contiguo (K10) se excavó parcialmente (subcuadros 4 a 9). Aquí sólo se documentó uno de los niveles, el nivel $\mathrm{B}$.

Dada la gran cantidad de restos arqueológicos recuperados en el proceso de excavación, en una primera fase de estudio del yacimiento se decidió analizar los materiales procedentes de muestra del cuadro K12, el único que conserva una estratigrafía completa. Dicha muestra, tomada al azar, se llevó a cabo en el subcuadro 2. Aquí procesamos, primero de forma independiente, cada una de las tallas excavadas (26 en total), separando los restos procedentes del cribado del material con cribas de 8, 4 y 2 mm de luz (ÁLVAREZ-FERNÁNDEZ et al., 2010a; 2013). Aunque también se utilizó la malla de $1 \mathrm{~mm}$, y a causa de la alta fragmentación, no se tuvo en cuenta en las contabilizaciones el número de restos (NR) documentado (miles), como tampoco se separaron diferentes partes que nos posibilitaban calcular el número mínimo de Individuos (NMI). Se establecieron las siguientes categorías generales: industria lítica, cerámica, ocre, carbón, hueso, moluscos marinos, crustáceos, equinodermos, y peces (Figura 2).
Posteriormente asignamos cada una de las tallas al nivel al que pertenecían. En el caso en el en una misma talla albergase materiales de dos niveles arqueológicos (base del nivel superior y techo del inferior), la información de los restos de dicha talla no se tenía en cuenta.

Por lo que se refiere a los restos de origen marino, siempre que se pudo, se identificaron a nivel de especie. La clasificación la hemos hecho a partir de sus caracteres taxonómicos (ornamentación, forma, etc.). Para ello hemos utilizado, por una parte, colecciones de referencia de moluscos, crustáceos y equinodermos, en el Departamento de Prehistoria, Historia Antigua y Arqueología de la Universidad de Salamanca. En el caso de los peces, sus restos han sido identificados de forma preliminar; todos sus elementos esqueléticos (huesos y dientes, o sus fragmentos) han sido incluidos en los recuentos.

La información sobre el hábitat de las especies ha sido tomada de diferentes estudios específicos (BORJA \& MUXICA, 2001; BORJA et al., 2004; CASTRO et al., 2006; IBAÑEZ 1982; IBAÑEZ \& FELIU 1983; MADINA, 2010: MORTENSEN, 1927; RELINI, 1980, SOUTHWARD \& CAMPBELL 2005, SOUTHWARD, 2008). Para los moluscos marinos, hemos seguido la sistemática de nomenclatura de CLEMAM ${ }^{1}$, mientras que para los crustáceos y equinodermos seguimos la de WORMS², y para los peces, la de ITIS $^{3}$.

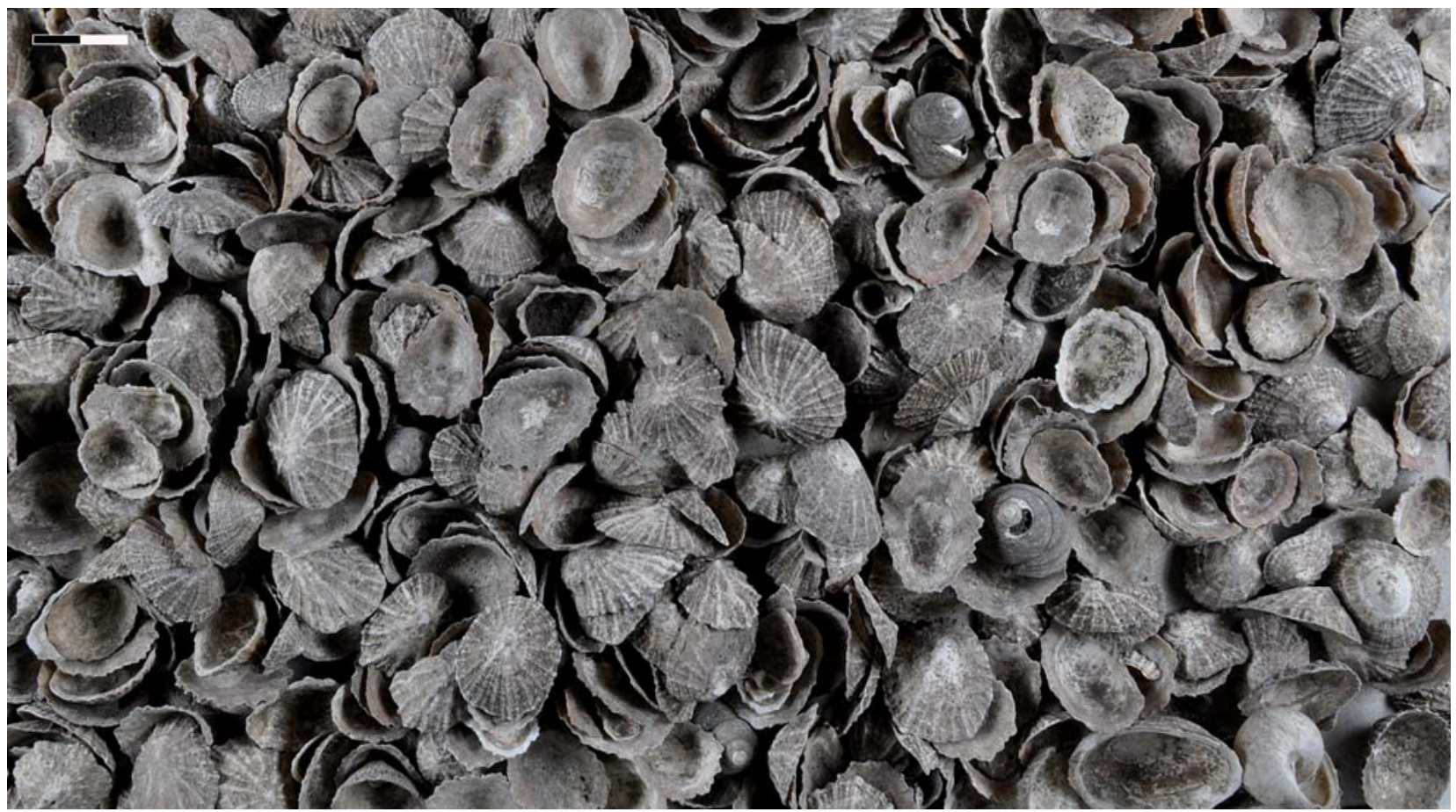

Fig. 2. J3. Material arqueomalacológico de la criba de $8 \mathrm{~mm}$. / J3. Archaeomalacological material in the $8 \mathrm{~mm}$ mesh screen.

${ }^{1}$ Check List of European Marine Mollusca, Muséum National d’Histoire Naturelle, Paris: Web Service available online at http://www.somali.asso.fr/clemam). ${ }^{2}$ World Register of Marine Species. Web Service available online at http://www.marinespecies.org) 20-12-2014 (Appeltans, et al., 2014).

${ }^{3}$ Integrated Taxonomic Information System, Web Service available online at http://www.itis.gov) 20-12-2014. 
En el caso de las conchas de moluscos, la metodología de cuantificación de estos restos ha sido tomada de MADARIAGA (1975) y de MORENO (1994), y adaptada a otros trabajos similares realizados por uno de nosotros en la Región Cantábrica (ver, por ejemplo, ÁLVAREZ-FERNÁNDEZ, 2007; 2012; 2013). Hemos tenido en cuenta el total del NR, sumando los separados en las cribas de 8, 4 y $2 \mathrm{~mm}$ de luz. De la criba de $2 \mathrm{~mm}$ solo hemos separado los ápices de los gasterópodos y las placas de terga, scuta, carina y rostra, completas y fragmentadas, de los percebes y balanos, ejemplares de $M$. neritoides, vértebras de pequeño tamaño y dientes de peces, etc. lo que ha posibilitado un aumento del NMI final (ÁLVAREZ-FERNÁNDEZ, et al. 2010a: 20 y ss. ). Además de estos restos identificados, se documentó una ingente cantidad de otros restos, fundamentalmente fragmentos de conchas de gasterópodos (sobre todo, lapas), fragmentos de placas de percebes, etc. La gran fragmentación y la mala conservación de gran parte de ellos, que impedía su determinación incluso a nivel de Filo, ha hecho que no sean incluidos en la contabilización total del NR.

El NMI se ha calculado con el fin de evitar una sobrerrepresentación de las especies más frágiles. Para el cálculo del NMI de los caracoles marinos se han tenido los restos con ápices íntegros (más abundantes que los restos con la zona umbilical conservada). Para las lapas se han cuantificado los restos con ápices no fragmentados. En el caso de los bivalvos se ha tenido en cuenta la charnela; las valvas derechas e izquierdas se suman por separado y se toma como NMI el número mayor (MORENO, 1994).

En el caso del percebe Pollicipes pollicipes (Gmelin, 1790), la metodología de cuantificación, tomada de ÁLVAREZ-FERNÁNDEZ et al. (2010b; 2013), tiene en cuenta las placas de mayor tamaño. En primer lugar se separan las terga (derechas e izquierdas), scuta (derechas e izquierdas), carina y rostra. Después se cuantifican. El NMI se estima a partir de la placa más abundante.

Por lo que se refiere a los balonomorfos, cuantificamos como especimen, cada concha entera (con o sin valvas operculares) o cada fragmento documentado (conjunto de placas y placas o valvas aisladas). También han sido cuantificadas como especimen las placas aisladas, tanto completas como fragmentadas. El género Chthamalus posee una base membranosa coronada por seis placas (una carena, dos laterales, dos rostro- laterales y un rostro) cerrado por cuatro valvas operculares (dos scuta y dos terga). El género Perforatus presenta una base calcificada y seis placas (una carena, dos careno-laterales, dos laterales, un rostro), cerrado por cuatro placas operculares. En ninguno de los niveles de J3 se han documentado balanos completos.

En los estudios sobre los erizos que se documentan en los sitios arqueológicos se tiene en cuenta la metodología de determinación y cuantificación propuesta por CAMPBELL (2008). Sin embargo, adelantamos que los restos de erizos de J3 son muy escasos (placas y espículas) y están muy fragmentados. Para identificar la especie a la que pertenecen se ha tomado en cuenta la cabeza de las púas, con ornamentación diferente según estemos ante una especie $u$ otra (ÁLVAREZ-FERNÁNDEZ, 2010: 91)

No hemos considerado el peso de los restos arqueológicos de origen marino, ni individual, ni colectivamente (ÁLVAREZ-FERNÁNDEZ, 2012). La precipitación de carbonato cálcico que presentan algunos de los restos aumentaría dicho peso, mientras que la descalcificación que presentan gran parte de ellos, lo disminuiría.

También se tuvieron en cuenta las alteraciones que poseían las distintas categorías establecidas. Así, se documentaron las provocadas por otros organismos marinos (epifauna) y las de tipo tafonómico. En estas últimas separamos aquellas en las que intervino la acción del hombre, de las provocadas por otros agentes.

Por último, se tomaron datos de tipo biométrico en ejemplares bien conservados (de conchas de moluscos) con el fin de establecer criterios que nos hablen de posibles cambios de talla en diferentes especies a lo largo de toda la secuencia del yacimiento, y poder así determinar a qué pudieron haber sido debidos (climáticos, antrópicos, etc.). Para ello nos hemos aplicado un Análisis de Varianza (ANOVA). Se complementa el análisis con una descripción gráfica mediante un Box plot.

Los restos ( $n=45200$ ) han sido estudiados en el Centro de Custodia de Materiales Arqueológicos y Paleontológicos del Gobierno Vasco en Gipuzkoa (San Sebastián).

\section{3.- RESULTADOS \\ 3.1. Composición faunística}

En todos los niveles de J3 predominan los restos de moluscos marinos (gasterópodos y bivalvos), con porcentajes que oscilan entre el 99,9\% del nivel $\mathrm{F}$ y el 95,8\% del nivel B. El porcentaje restante lo forman placas de percebe, placas e individuos completos balanos, placas y púas de erizo y dientes, vértebras, y otros huesos de peces (Tablas 1 y 2).

Por lo que se refiere a los moluscos, teniendo en cuenta el NMI de cada nivel observamos que, salvo a un escaso número de bivalvos (mejillón y almeja), prácticamente la totalidad de los individuos de los cuatro niveles de conchero está formada por gasterópodos (Tabla 2; Figura 3). El género Patella supone entre el $94,8 \%$ (nivel D) y el 98,1\% (nivel B). Phorcus lineatus está presente en los cuatro niveles, aunque sólo en el nivel D llega a alcanzar el $5 \%$. El resto, lo forman escasos restos de otras especies de gasterópodos (Stramonita haemastoma, Haliotis tuberculata tuberculata, Melarhaphe neritoides y Gibbula sp.).

Una gran parte de las lapas de los cuatro niveles de J3 no ha podido ser determinada a nivel de especie, debido a la mala conservación de sus conchas (entre el ca 


\begin{tabular}{|l|r|r|r|r|r|r|}
\hline & Moluscos & Percebes & Balanomorpha & Erizos & Peces & TOTALES \\
NIVEL B & 7984 & 329 & 28 & 0 & 1 & 8342 \\
\hline NIVEL C & 4371 & 134 & 50 & 3 & 9 & 4567 \\
\hline NIVEL D & 21701 & 67 & 127 & 18 & 17 & 21930 \\
\hline NIVEL F & 10357 & 0 & 0 & 0 & 4 & 10361 \\
\hline & 44413 & 530 & 205 & 21 & 31 & $\mathbf{4 5 2 0 0}$ \\
\hline
\end{tabular}

Tabla 1: NR de animales de origen marino en los diferentes niveles de $\mathrm{J} 3$ (muestra de K12, Sc2).

Table 1: NR of marine animals in the different levels at $\mathrm{J} 3$ (sample from K12, sector 2).

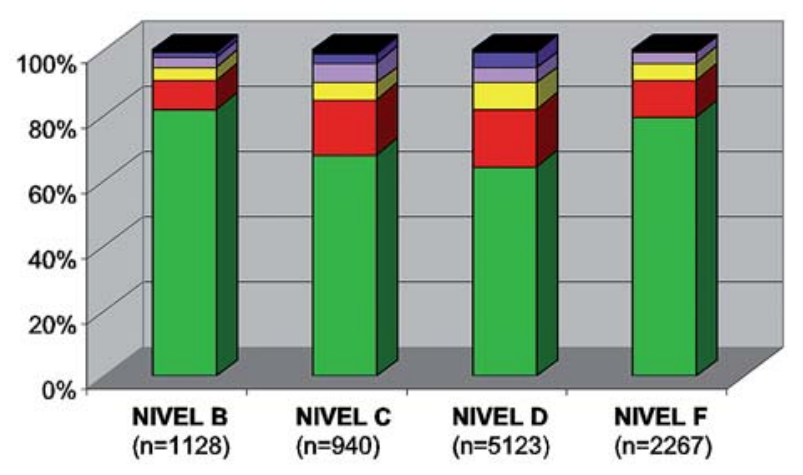

motros $\square$ P. lineatus $\square P$. ulyssiponensis $\square P$. vulgata $\square P$. depressa $\square$ Patella sp.

Fig. 3. Porcentajes de las diferentes especies de moluscos marinos en los diferentes niveles de J3 (muestra de K12, Sc2), según el NMI. / Percentages of the marine mollusc species in the different levels at $\mathrm{J} 3$ (sample from $\mathrm{K} 12$, Sector 2), according to the MNI.

$68 \%$ en el nivel C y ca $84 \%$ del nivel B). Aún así, se han podido clasificar tres especies en los cuatro niveles: Patella vulgata, Patella depressa y Patella ulyssiponensis. En la secuencia predomina $P$. depressa, con porcentajes entre ca 54\% (nivel B) y ca 60,5\% (nivel C). Salvo en el nivel C, la segunda especie representada es $P$. vulgata, con porcentajes cercanos al 26\%. P. ulyssiponensis alcanza el 21,6\% en el nivel C (Figura 4).

Por lo que se refiere a los crustáceos, se han documentado placas de percebe y ejemplares completos y fragmentados de balanos.

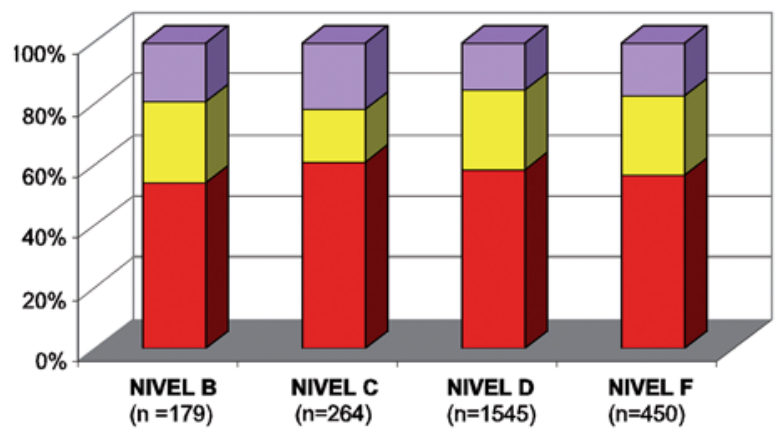

$\square \boldsymbol{P}$. ulyssiponensis $\square \boldsymbol{P}$. vulgata $\square \boldsymbol{P}$.depressa

Fig. 4. Porcentajes de las diferentes especies de lapas en los diferentes niveles de J3 (muestra de K12, Sc2), según el NMI. / Percentages of the limpet species in the different levels at J3 (sample from K12, Sector 2), according to the MNI

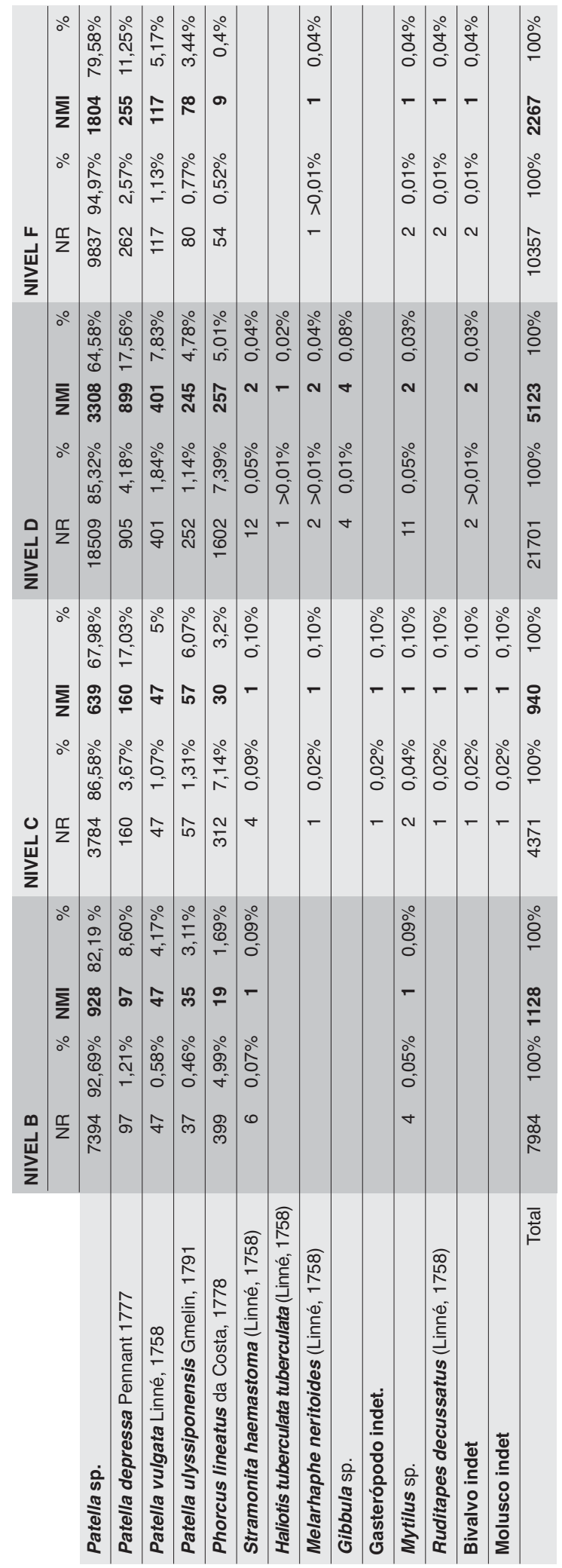

Tabla 2: Distribución absoluta y porcentual de los taxones de los niveles arqueológicos documentados en J3 (niveles B, C, D y F). NR: Número de restos; NMI: Número Mínimo de Individuos.

Table 2: Absolute and percentage distribution of the taxa in the archaeological levels documented at J3 (Levels B, C, D and F). NR: Number of remains; MNI: Minimum Number of Individuals. 
Se han recuperado placas de percebe de gran tamaño (terga, scuta, carina, rostra), pero también otras placas menores, estas últimas procedentes en su mayoría del cribado del sedimento con la mallas de 4 y $2 \mathrm{~mm}$ de luz (Figura 5). El nivel $F$ es el único nivel en el que no se documentaron restos de este crustáceo. A partir de las placas de mayor tamaño hemos determinado un NMI de 58 para el nivel B, de 19 para el nivel C y 14 para el nivel D. Los individuos de los niveles B y D han sido contabilizados a partir de placas completas y fragmentos proximales de placa de Carina y los del nivel C, a partir de placas completas y fragmentos proximales de placas derechas de scuta.

Por lo que se refiere a los balanomorfos, se han tenido en cuenta las conchas completas e incompletas, que se documentaron adheridas a la parte externa de algunas lapas y sueltas en el sedimento de los niveles. Los especímenes pertenecen a Chthamalus. El estudio de algunas de las conchas enteras (6 placas unidas, una de ellas con placas operculares) nos ha permitido determinar las especies Chthamalus montagui Southward, 1976 (nivel D) y Chthamalus stellatus (Poli, 1795) (Nivel C). Algunos ejemplares de Perforatus perforatus perforatus (Bruguière, 1789) proceden del nivel C/D (no cuantificados en la Tabla 1, al proceder del contacto entre dos niveles) y del nivel C (Figura 6).

Por lo que se refiere a los equinodermos, se han documentado pequeños fragmentos de caparazón y fragmentos de púas. Las bases de las púas de los erizos poseen pequeños tubérculos a partir de los que salen las estriaciones características. En la especie intermareal $\mathrm{Pa}$ racentrotus lividus (Lamarck, 1816) estos tubérculos poseen forma de alubia. Esta característica es diferente en otras especies intermareales que habitan el cantábrico, como Psammechinus miliaris (P.L.S. Müller, 1771). Esta última especie citada presenta en la bases de las púas, tubérculos en forma de pequeñas cúpulas (Figura 7). Los restos de este equinodermo sólo están presentes en los niveles C y D. Se estima un ejemplar por nivel.

Por último, los restos de peces examinados son, al igual que los de los erizos, muy escasos representando menos del 1\% del material analizado en el Subcuadro 2 ( $N R=31 ; 0,06 \%)$, encontrándose documentado en los cuatro niveles del yacimiento: Nivel $\mathrm{B}(\mathrm{NR}=1)$, Nivel $\mathrm{C}(\mathrm{NR}=$ 9), Nivel D (NR=17), y Nivel F $(N R=4)$ (Tabla 1). Entre los elementos esqueléticos reconocibles se cuentan sobre todo dientes sueltos (10), vértebras (8), y un fragmento de hueso mandibular (1), que documentan la presencia de peces de la familia Sparidae. Tres de los dientes analizados corresponden, al género Diplodus Rafinesque, 1810, tratandose posiblemente de Diplodus sargus (Linné, 1758).

Los Sargos (Diplodus spp.) son peces costeros que ocupan praderas submarinas y facies rocosas, sobre todo las revestidas de mejillones, que trituran fácilmente gracias a sus molares. El sargo Diplodus sargus también se come a los erizos (BAUCHOT \& PRAS, 1993).

\subsection{Conservación y alteraciones}

El estado conservación de gran parte de material arqueofaunístico de los diferentes niveles de J3 es relativamente aceptable.

Los moluscos poseen una serie de alteraciones provocadas por organismos marinos (epifauna), antes de que fueran recogidos por los grupos humanos. Los restos también aparecen alterados por la acción del hombre (fuego, pisoteo), pero, sobre todo, a causa de estar continuamente expuestos a la intemperie, particularmente, por el agua de la lluvia.

Por lo que se refiere a la epifauna, en la superficie de algunas de las lapas se han documentado balanos. 21 de

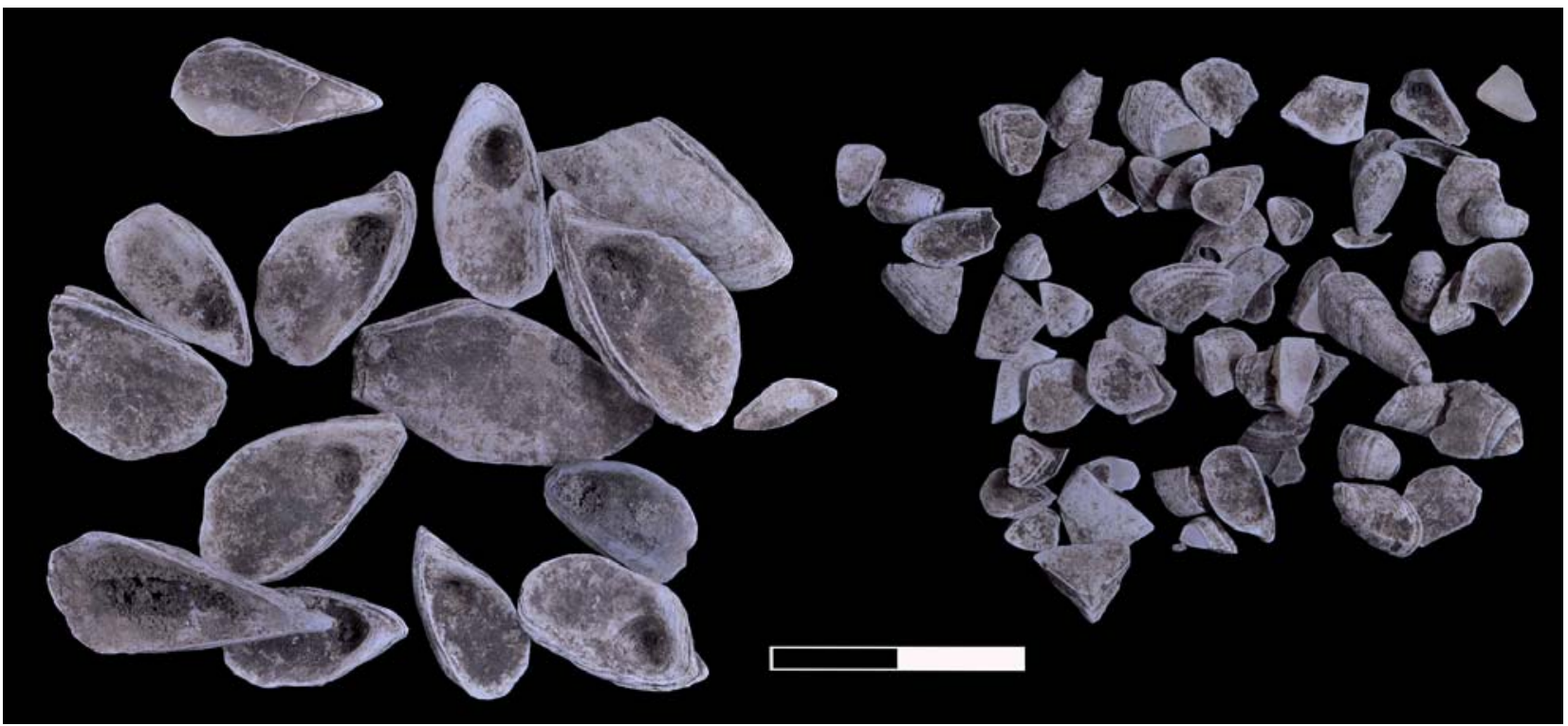

Fig. 5. Placas depercebes (Pollicipes pollicipes) de J3 (nivel B), a la izquierda, recogidos con la malla de $8 \mathrm{~mm}$, a la derecha con la malla de $2 \mathrm{~mm}$. / Goose barnacle (Pollicipes pollicipes) plates at J3 (Level B), on the left in an $8 \mathrm{~mm}$ mesh screen and on the right in a $2 \mathrm{~mm}$ mesh screen. 


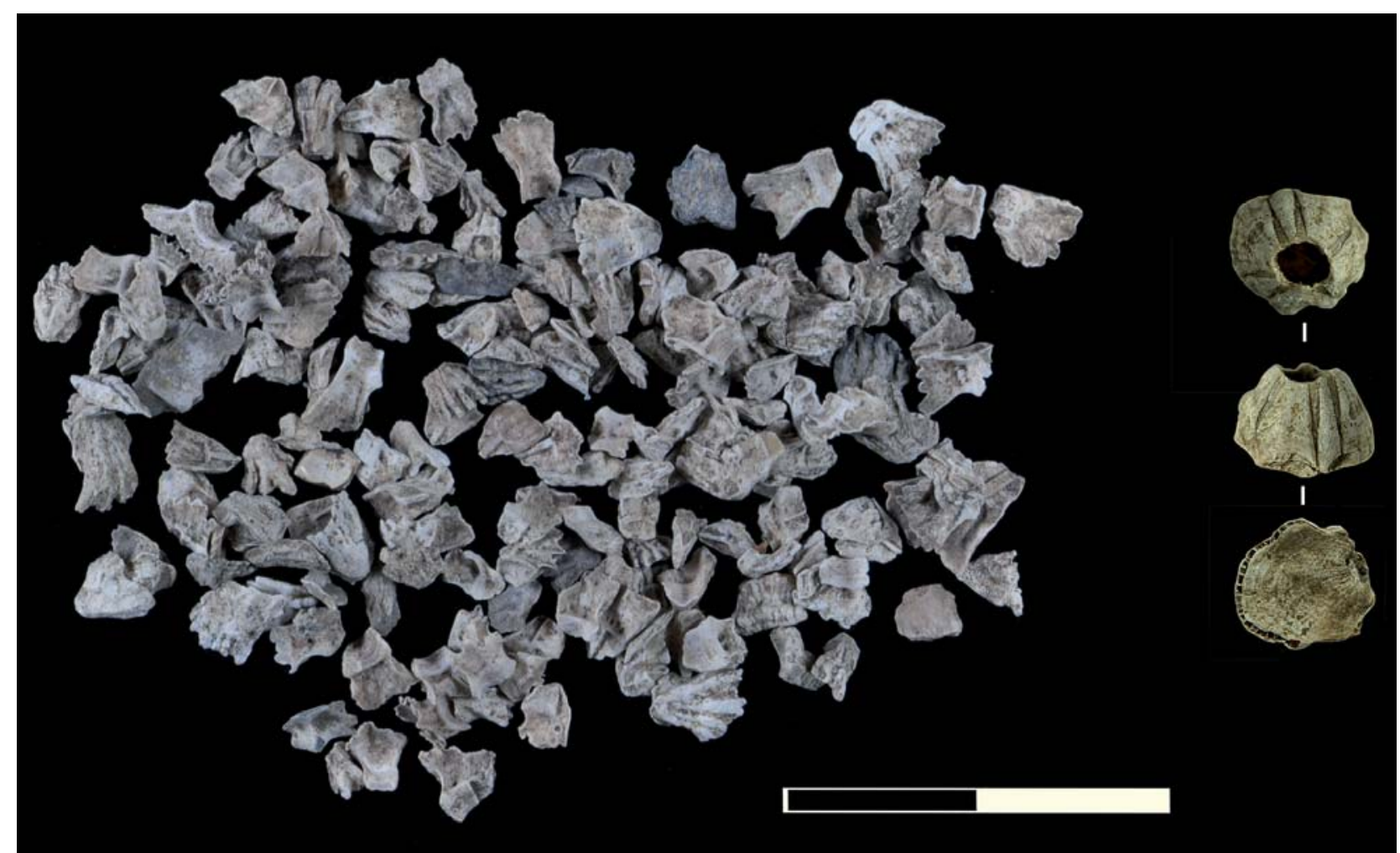

Fig. 6. Placas de Chthamalus sp. (nivel D) y ejemplar de (nivel C/D) de J3. / Chthamalus sp. plates (Level D) and Perforatus perforatus perforatus specimen (Level C/D) at J3.

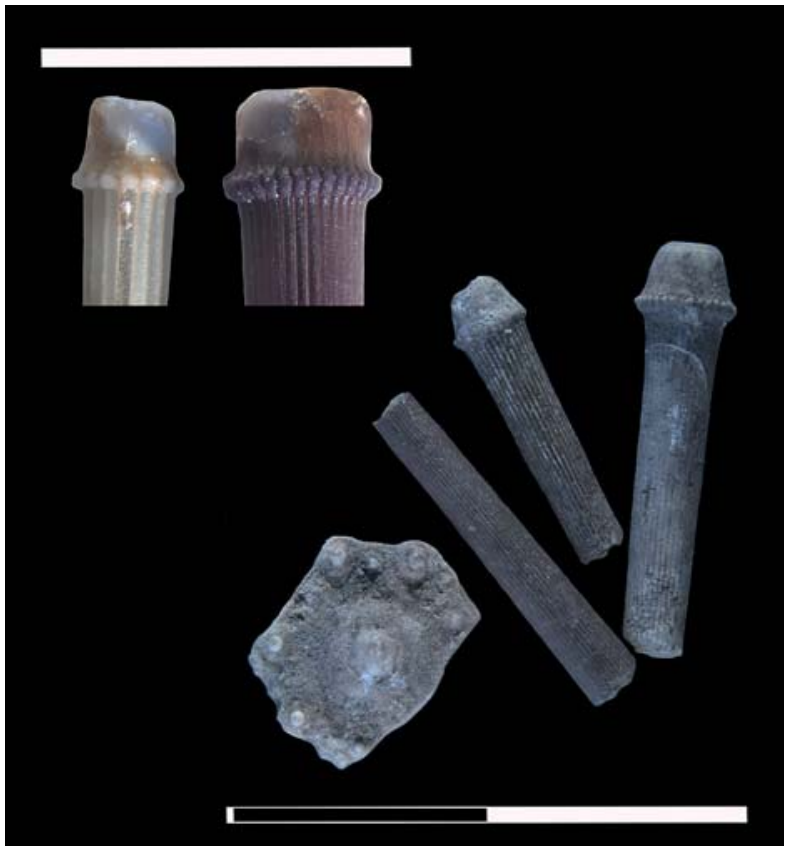

Fig. 7. Arriba: espículas de erizos actuales de Paracentrotus lividus (derecha) y Psammechinus miliaris (izquierda). Abajo: Fragmentos de caparazón y púas de Paracentrotus lividus de J3 (nivel D). / Top: spicules of modern sea urchins: Paracentrotus lividus (right) and Psammechinus miliaris (left). Bottom: Paracentrotus lividus carapace fragments and spines from J3 (Level D).
$P$. ulyssiponensis y una $P$. depressa en el nivel C, y dos $P$. ulyssiponensis y tres $P$. depressa en el nivel $\mathrm{D}$, conservan uno o varios Chthamalus. La gran cantidad de restos de balanos documentados sueltos en el sedimento puede ser explicada, porque éstos vinieron adheridos a los moluscos traídos como alimento. Posteriormente, se desprendieron de las superficies de las conchas que colonizadas.

El hombre, como agente tafonómico, ha actuado en los restos faunísticos. Así, algunas de las lapas mejor conservadas poseen una parte de sus bordes rotos. Estas roturas posiblemente fueron provocadas al intentar separar estos gasterópodos de las rocas con algún tipo de útil, lítico u óseo.

En líneas generales, podemos indicar que el porcentaje de los individuos quemados no es muy elevado en los moluscos más abundantes, las lapas (<25\%). Sí es elevado en $P$. lineatus, pero sólo en los dos niveles más recientes (niveles B y C). El NMI de esta especie muy bajo en los dos niveles (Nivel B = 19; nivel C =30) (Tabla 3).

El hombre también fragmentó los restos de las conchas, posiblemente mediante el pisoteo. Esta fragmentación es alta en los cuatro niveles. Centrándonos en el género Patella, y si aplicamos el Índice de Fragmentación (ÁLVAREZ-FERNÁNDEZ, 2007) observamos que el nivel B, el más reciente, es el que está más fragmentado (Tabla 4).

A causa de la escasa cobertura del abrigo, el yacimiento ha estado expuesto durante su formación y hasta la 


\begin{tabular}{|l|r|r|r|}
\hline & Patella sp. & \multicolumn{1}{|c|}{ P. lineatus } & P. pollicipes \\
\hline NIVEL B & $23,12 \%$ & $84,21 \%$ & $34,48 \%$ \\
\hline NIVEL C & $24,36 \%$ & $80 \%$ & $26,31 \%$ \\
\hline NIVEL D & $18,07 \%$ & $14,39 \%$ & $21,42 \%$ \\
\hline NIVEL F & $15,79 \%$ & $22,22 \%$ & \\
\hline
\end{tabular}

Tabla 3: Porcentaje los invertebrados más abundantes que aparecen quemados en los 4 niveles de $\mathrm{J} 3$.

Table 3: Percentage of the most abundant invertebrates displaying signs of burning in the four levels at $\mathrm{J} 3$.

\begin{tabular}{|l|r|r|r|r|}
\hline & NIVEL B & NIVEL C & NIVEL D & NIVEL F \\
\hline (NR / NMI) / 100 & 0,068 & 0,044 & 0,045 & 0,045 \\
\hline (NR /N ${ }^{\circ}$ de ejemplares enteros)/100 & 0,587 & 0,337 & 0,192 & 0,296 \\
\hline
\end{tabular}

Tabla 4: Índice de fragmentación de Patella sp de los 4 niveles de $\mathrm{J} 3$ : (Número de Restos / Número Mínimo de Individuos) / 100) y (Número de Restos / Número de los ejemplares enteros) /100)

Table 4: Fragmentation indices for Patella sp. in the four levels at $\mathrm{J} 3$ (Number of Remains/Minimum Number of Individuals/100) and (Number of Remains/Number of whole specimens/100).

actualidad a las inclemencias meteorológicas. El agente tafonómico que ha afectado más a los restos arqueológicos ha sido la acción del agua de la lluvia. Su bajo Ph ha deteriorado, en mayor o menor medida, los restos faunísticos. Esto afecta principalmente a los restos óseos del individuo inhumado del nivel $\mathrm{D}$, así como de otros mamíferos de los niveles B, C, D y F. Estos restos aparecen con alto grado de corrosión (descalcificación), lo que facilitó además su mayor fragmentación (IRIARTE et al., 2005). También afectó a los restos de organismos marinos, sobre todo a las conchas de las lapas; gran parte carece de coloración interna, lo que imposibilita su identificación a nivel de especie. Así, y teniendo en cuenta las lapas completas y fragmentadas (con el ápice completo conservado), el porcentaje de los individuos que hemos determinado a nivel de especie ( $P$. vulgata, $P$. depressa y $P$. ulyssiponensis) varía entre 15\%, en el nivel $B$ y el $32 \%$ en el nivel D. Además, las superficies de gran parte de las lapas, sobre todo del nivel F, aparecen pulverulentas y con un color blanquecino. Finalmente, el porcentaje de los restos con precipitación de carbonato cálcico es muy bajo en los cuatro niveles ( $<1 \%$ de los restos).

Hay que señalar que, los componentes carbonatados de las lapas han protegido a los restos de invertebrados de pequeño tamaño, conservándolos en su interior. De esta forma se explicaría la existencia de restos que, de otro modo hubiesen desaparecido, por ejemplo dientes de sargo, las conchas completas de $M$. neritoides y las placas de percebe y de diferentes especies de balanos.

\subsection{Biometria}

Dada la gran cantidad de restos de animales documentados, J3 es un yacimiento ideal para realizar análisis biométricos y comparar los resultados procedentes de sus cuatro niveles arqueológicos. Hasta el momento, hemos realizado este tipo de mediciones en moluscos, crustáceos y peces procedentes del subcuadro 2 del cuadro K12. La valoración de los tamaños de las placas de los percebes ha sido recientemente presentada (ÁLVAREZ-FERNÁNDEZ et al., 2013). La que presentamos aquí se refiere a las conchas del genero Patella. En la figura 8 se presentan los box-plot por niveles, donde puede observarse que tanto la mediana como el rango intercuartílico (longitud de la caja) es muy similar en los diámetros máximos de las lapas de los cuatro niveles, tanto en amplitud como en valores, si bien es el nivel $D$ el que presenta una variabilidad ligeramente superior. Se ha realizado un ANOVA (Análisis de la varianza) cuyos resultados ponen de manifiesto que no hay diferencias significativas entre los diámetros medios de los ejemplares pertenecientes a cada uno de los cuatro niveles $(p$-valor $=0.146)$.

\section{4.- DISCUSIÓN}

El abrigo de J3 es uno de los pocos concheros estratificados documentados en la Región Cantábrica. El que posea dos niveles (niveles F y D) datados en el Mesolítico y otros dos adscritos a periodos posteriores (niveles $\mathrm{C} \mathrm{y}$ $\mathrm{B})$, hacen que sea un yacimiento clave para comprender el papel que jugaron los recursos marinos en la alimentación de los últimos grupos de cazadores-recolectores y primeros productores de alimentos en la región.

La investigación que presentamos en este artículo, si bien es preliminar (calculamos que sólo se ha estudiado el $<7 \%$ del total de evidencias documentadas en el proceso de excavación), nos permite ofrecer los primeros datos referentes a su composición, a las zonas de recolección de los animales en la costa y a las alteraciones de los restos.

La composición arqueofaunística es similar a lo largo de toda la secuencia de J3. Los moluscos son los animales marinos más abundantes en los custro niveles de conchas del yacimiento, con porcentajes de entre cerca del 95\% (nivel B) y el 99,9\% (nivel F), según el NR.

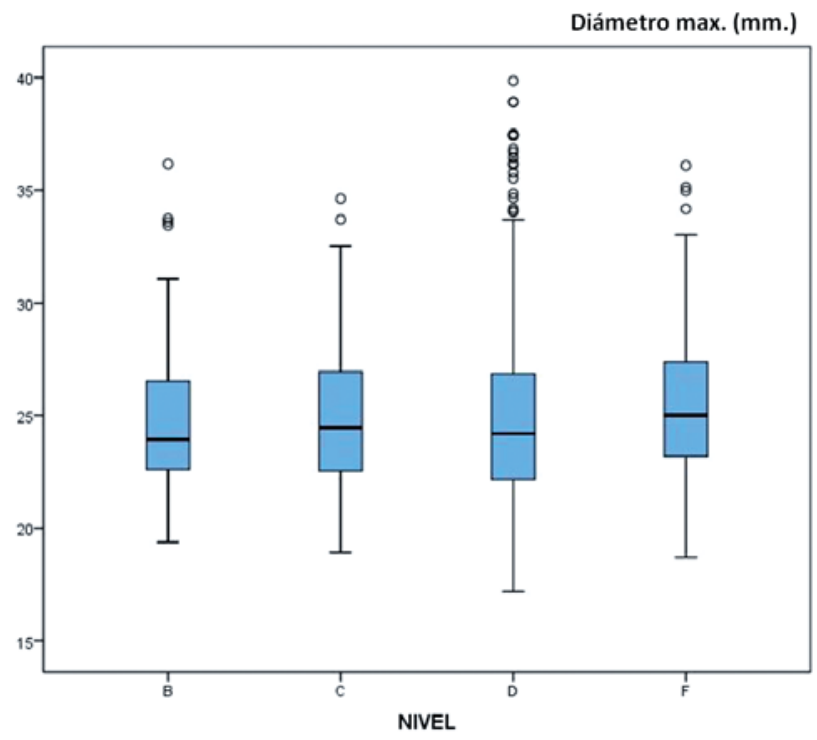

Fig. 8. J3. Boxplot de los diámetro máximos (en $\mathrm{mm}$ ) de ejemplares del género Patella ( $P$. depressa $+P$. vulgata $+P$. ulyssiponensis) procedentes de los niveles $\mathrm{B}(n=70), \mathrm{C}(n=106), \mathrm{D}(n=739)$ y $\mathrm{F}(n=199)$. / Boxplot with the maximum diameters (in $\mathrm{mm}$ ) of Patella $(P$. depressa $+P$. vulgata $+P$. ulyssiponensis) specimens in Levels $\mathrm{B}(n=70), \mathrm{C}(n=106), \mathrm{D}(n=739)$ and $\mathrm{F}(n=199)$. 
En los cuatro niveles predominan las lapas, con porcentajes >95\% (según el NMI). Un porcentaje pequeño de estos gasterópodos ha podido ser determinado a nivel de especie. $P$. depressa es la más abundante, pero también se han clasificado ejemplares, tanto de $P$. vulgata, como de $P$. ulyssiponensis. El caracol $P$. lineatus está presente en toda la secuencia, pero es poco abundante. El resto de moluscos documentados (los gasterópodos Stramonita haemastoma, H. tuberculata tuberculata y Gibbula sp., los bivalvos Mytilus sp. y $R$. decussatus) son muy escasos. También está muy poco representado el erizo P. lividus. Dados los escasos restos de estas últimas especies citadas, consideramos que los grupos humanos no las recogieron como alimento, sino que su presencia en el yacimiento se deba a otros motivos, como curiosidades, traídas en los estómagos de otros animales (por ejemplo, los peces) consumidos en el yacimiento, etc.

Entre los crustáceos, sólo los percebes fueron recogidos como alimento. Sólo están presentes en los niveles más recientes, B, C y D. El porcentaje es siempre $<5 \%$ de los invertebrados.

Los restos de peces marinos se documentaron en los cuatro niveles, pero son comparativamente escasos. Las marcas de fuego registradas en algunos de los restos analizados señala la acción humana. La ausencia de elementos deformados, y el mismo tamaño de los huesos analizados en el subcuadro 2 (correspondientes a peces de talla mediana) permiten indicar igualmente que fueron pescados por grupos humanos.

A tenor de las especies documentadas, podemos afirmar que posiblemente los grupos humanos marisqueaban sobre las rocas desprendidas del acantilado rocoso situado $200 \mathrm{~m}$ del abrigo. Las zonas que serían explotadas irían desde el intermareal superior (zona que se cubre con las mareas medias diarias) hasta la zona intermareal inferior (sólo descubierta en los periodos de mareas vivas). Esta parece ser la tónica general de explotación de los recursos marinos en la Región Cantábrica a comienzos del Holoceno, tanto durante el Mesolítico y el Neolítico (ÁLVAREZ-FERNÁNDEZ, 2011; e. p.; ÁLVAREZ-FERNÁNDEZ et al., 2011; GUTIÉRREZ, 2009), como en periodos posteriores (Calcolítico y Edad de Bronce) ÁLVAREZ-FERNÁNDEZ, e. p.; ÁLVAREZ-FERNÁNDEZ, et al., 2014)

Todas las especies de animales marinos documentadas en J3 son especies que habitan en la actualidad en las costas guipuzcoanas. P. vulgata habita desde el intermareal superior hasta el intermareal inferior. $P$. aspera se encuentra en las pozas tidales del intermareal superior, y sobre las rocas del intermareal medio (franja que se descubre parcialmente durante la marea baja) y en menor medida en el intermareal inferior. P. ulyssiponensis habita en el intermareal medio y sobre todo, en el intermareal inferior. Los caracoles $P$. lineatus se localizan en las zonas intermareal superior y media. Por último, las piñas del percebe serían recogidas a pie sobre rocas y en sus grietas, en el intermareal inferior.
La presencia de otros animales, carentes de interés alimenticio para los grupos prehistóricos precisaría igualmente las zonas de recolección de moluscos como alimento. Melaraphe neritoides, especie de pequeño tamaño que vive sobre las rocas y sobre otros gasterópodos, apoyaría el hecho de la recogida de las lapas y de $P$. lineatus en la zona intermareal superior y media. Lo mismo ocurriría con los balanomorfos pegados a la superficie externa de las lapas. Así, Chthamalus habita sobre todo en el intermareal; generalmente $C$. montagui habita la zona superior y $C$. stellatus, la media y la inferior. $P$. perforatus perforatus habita en el intermareal inferior y puede llegar a colonizar la zona infralitoral.

Particularmente interesante resulta la presencia de S. haemastoma (Figura 9), gasterópodo que habita en la zona intermareal y los primeros metros del infralitoral. En la actualidad, es considerado en la costa vasca como "especie invasora" (MARTÍNEZ \& ADARRAGA 2006). Las conchas de esta especie se ha documentado, además de en tres de los niveles de J3, en otros yacimientos vascos desde finales del último periodo glaciar. Así, se citan cuatro restos en el Gravetiense final/ Solutrense (nivel II) de Aitzbitarte III-entrada (Rentería, Gipuzkoa) (ÁLVAREZ-FERNÁNDEZ, 2011b). También está presente en el Magdaleniense superior (nivel D) de Urtiaga (Itziar, Gipuzkoa) (BARANDIARÁN, 1947: 441) y en el yacimiento mesolítico de Kobeaga II (Ispaster, Bizkaia) (MADARIAGA DE LA CAMPA, 1976: 206). Si esta especie habitaba las costas del País Vasco en la Prehistoria, o si sus conchas han llegado por medio de contactos a larga distancia con grupos que habitaban las costas mediterráneas, es algo que debe precisarse en el futuro.

Por lo que se refiere a las alteraciones de los restos, aparte de las de tipo tafonómico ligadas a la acción del agua de la lluvia después de haberse formado las acumulaciones de conchas, hay que destacar aquellas provocadas por la acción del hombre, sobre todo la fragmentación por pisoteo y el fuego. En cuanto a este último, hay que indicar que en la pequeña zona del yacimiento excavado (que está al lado del abrigo) no se documentaron evidencias de hogares, por lo que esta zona marginal quizá funcionó como basurero. Esta interpretación sería válida para los cuatro niveles con conchero de J3, incluido el nivel $\mathrm{D}$, donde además se localizó el enterramiento de un individuo datado en el Mesolítico.

Por último, debemos indicar que los primeros datos biométricos sobre Patella sp., así como de los percebes, indican que la talla de este gasterópodo resulta similar en toda la secuencia de J3, por lo que no se observa ningún indicio que nos indique una hipotética sobre-explotación del medio marino.

El análisis del resto de evidencias faunísticas procedentes de otros subcuadros de la excavación de J3 permitirá, en un futuro próximo, precisar las observaciones que se incluyen en esta presentación preliminar sobre los recursos de origen marino del yacimiento. 


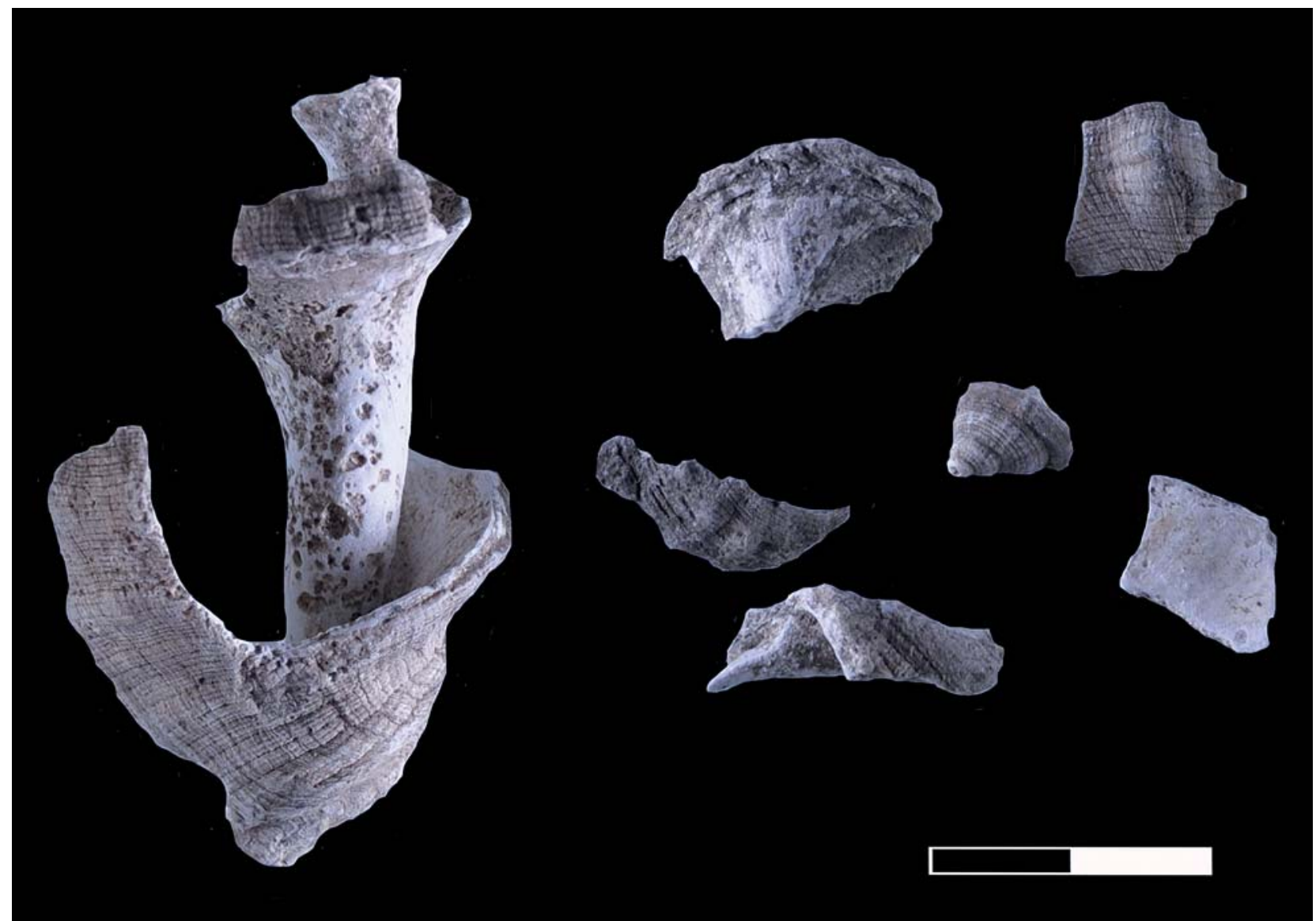

Fig. 9. Fragmentos de Stramonita haemastoma de J3 (nivel D). / Stramonita haemastoma fragments in Level D at J3.

\section{5.- AGRADECIMIENTOS}

Agradecemos al Centro de Custodia de Materiales Arqueológicos y Paleontológicos en Gipuzkoa las facilidades que nos dieron para el estudio de los materiales arqueofaunísticos. También agradecemos a Jesús Altuna, Nathalie Desse-Berset, Yves Gruet y Didier Nereadeau la ayuda prestada en la determinación de alguno de los restos arqueofaunísticos de J3 y a Miriam Cubas, Marián Cueto Catherine Dupont y Jesús Tapia por las discusiones que hemos tenido sobre diversos aspectos del yacimiento. Esta investigación ha sido financiada por los siguientes proyectos: Los recursos marinos en los yacimientos prehistóricos de Europa Occidental, financiado por el Ministerio de Ciencia e Innovación, Subprograma Ramón y Cajal (RYC-2009-04668); Catalogación de los restos arqueofaunísticos de origen marino de los yacimientos arqueológicos del País Vasco, financiado por el Departamento de Cultura del Gobierno Vasco, La explotación de los recursos marinos en la Europa Atlántica durante el Pleistoceno tardío y el Holoceno, financiado por el Ministerio de Economía y Competividad. Plan Nacional de I+D+I (HAR2011-29907-C03-03/HIST) (Investigador principal: E. Álvarez-Fernández) y por el Grupo de investigación consolidado en Prehistoria (IT-622-13) de la Universidad del País Vasco.

\section{BIBLIOGRAFÍA}

\section{ÁLVAREZ-FERNÁNDEZ, E.}

2007 La explotación de los moluscos marinos en la Cornisa Cantábrica durante el Gravetiense: primeros datos de los niveles E y F de La Garma A (Omoño, Cantabria). Zephyrus, LX 43-58.

2010 Moluscos, crustáceos y equinodermos: restos de origen marino en los yacimientos arqueológicos. El Futuro del Pasado, 1, 83-95.

2011a Humans and marine resource interaction reappraised: archaeofauna remains during the Late Pleistocene and Holocene in Cantabrian Spain. Journal of Anthropological Archaeology, 30 (3), 327-343.

2011b Estudio de los moluscos y los crustáceos de Aitzbitarte III (Zona de la entrada). En: ALTUNA J., et al. (Eds.): Ocupaciones humanas en Aitzbitarte III (País Vasco). 33.60018.400 (Zona de entrada a la cueva): 518-528. EKOB 5, Colección de Patrimonio Cultural Vasco, Servicio Central de Publicaciones del Gobierno Vasco, Vitoria.

2012 Investigaciones arqueomalacológicas en la Garma A (Omoño, Cantabria): los moluscos marinos de los niveles N y O (Magdaleniense superior). En: ARIAS, P., et al. (eds.) El Paleolítico superior cantábrico: 145-155. Publican, Universidad de Cantabria (IIIPC, Monografías 3), Santander.

2013 Upper Pleistocene-early Holocene transition at La Garma A cave (Omoño, Cantabria, Spain): first information about the Marine Molluscs. In: BAILEY, G. N. et al. (eds.) Shell 
Energy. Molluscs Shells as Coastal Resources: 167-181. Oxbow, Oxford.

e.p. Continuity of Human Marine Fauna Interaction during the Holocene in Cantabrian Spain. Quaternary International.

ÁLVAREZ-FERNÁNDEZ, E.; ALTUNA, J.; BARRERA-MELLADO, I.; CUBAS, M.; FERNÁNDEZ-GÓMEZ, M. J.; FERNÁNDEZ, R.; GRUET, Y.; MARIEZKURRENA, K. y ONTAÑÓN, R.

2014 Evolution de l'exploitation des ressources animales dans la région cantabrique entre 4500 et 2000 cal BC: la grotte de Los Gitanos (Cantabrie, Espagne). Comptes Rendus Palevol, 13, 307-316.

ÁLVAREZ-FERNÁNDEZ, E.; BARRERA, I.; BORJA, A.; FERNÁNDEZ, M. J.; IRIARTE, M. J. y ARRIZABALAGA, A.

2013 Biometric analysis of the stalked barnacle Pollicipes pollicipes from a Holocene archaeological site in the Jaizkibe Mountains (Gipuzkoa, Basque Country, Northern Spain). The Holocene, 23 (10), 1373-1380.

ÁLVAREZ-FERNÁNDEZ, E., CHAUVIN, A., CUBAS, M., ARIAS, P. y ONTAÑÓN, R.

2011 Mollusc Shell sizes in Archaeological contexts in Northern Spain (13,200 to 2,600 Cal BC): new data from La Garma A and Los Gitanos (Cantabria). Archaeometry, 53 (5), 963-985.

ÁLVAREZ-FERNÁNDEZ, E., IRIARTE, M.J. y ARRIZABALAGA, A. 2010a J3 (Hondarribia, Guipúzcoa): consideraciones de tipo metodológico y primeros resultados sobre los recursos marinos de un conchero de época mesolítica. En: GONZALEZ, E., et al. (Eds.) I. Reunión Científica de Arqueomalacología de la Península Ibérica: 17-24. Férvedes 6. Villalba.

\section{ÁLVAREZ-FERNÁNDEZ, E., ONTAÑÓN, R. y MOLARES, J.}

2010b Archaeological data on the exploitation of the gooseneck barnacle Pollicipes pollicipes (Gmelin, 1790) in Europe. Journal of Archaeological Science, 37 (2), 402-408.

APPELTANS, W., BOUCHET, P., BOXSHALL, G.A., DE BROYER, C., DE VOOGD, N.J., GORDON, D.P., HOEKSEMA, B.W., HORTON, T., KENNEDY, M., MEES, J., POORE, G.C.B., READ, G., STÖHR, S., WALTER, T.C. y COSTELLO, M.J. (eds)

2014 World Register of Marine Species. Accessed at http://www.marinespecies.org (2014-02-19).

ARIAS, P., FERNÁNDEZ-TRESGUERRES, J.A., ÁLVAREZ-FERNÁNDEZ, E., ARMENDARIZ, A., CUETO, M., FANO, M.A., FERNÁNDEZ, R., GARRALDA, M.D., MENSUA, C. y TEIRA L.C.

2007 Excavación arqueológica de urgencia en la cueva de La Poza I’Egua (Lledías, Llanes). Excavaciones Arqueológicas en Asturias, V Oviedo: 227-239.

BAUCHOT, M.L. y PRAS, A.

1993 Guía de los peces de mar de España y de Europa. Omega, Barcelona.

\section{BARANDIARÁN, J.M.}

1947 Exploración en la cueva de Urtiaga (en Itziar, Guipúzcoa). Guernika Eusko-Jakintza Revue des Etudes Basques, Bayona, I, 679-696.
BORJA, A., MUXIKA, I.

2001 Actualización del catálogo de los moluscos marinos de la costa vasca, en campañas realizadas por AZTI. Iberus, 19 (I), 67-85

BORJA, A., AGUIRREZABALAGA, F., MARTíNEZ, J., SOLA, J.C., GARCÍA ARBERAS, L. y GOROSTIAGA, J.M.

2004 Benthic communities, biogeographiy and ressources management. En: BORJA, A. y COLLINS, M. (eds.) Oceanography and Marine Environment of the Basque Country: 455-492. Elsevier Oceanography Series 70, Oxford.

\section{CAMPBELL, G}

2008 Sorry, wrong phylum: a neophyte archaeomalacologist's experiences in analyzing a European Atlantic Sea urchin assemblage. Archaeofauna, 17, 77-90

CASTRO, R., URIARTE, A., FRANCO, J., URIARTE, A., BORJA, A., GONZÁLEZ, M., VALENCIA, V., QUINCOCES, I., SOLLAUN, O. y GALPAROSO, 1 .

2006 Guía de la biodiversidad marina del Golfo de Bizkaia. Gobierno Vasco (Biodiversidad 5). Vitoria.

FANO, M.A

2007 Un nuevo tiempo: El Mesolítico en la región cantábrica. En: FANO, M. A. (ed.) Las Sociedades del Paleolítico en la Región Cantábrica: 337-402. Diputación Foral de Bizkaia (Kobie, anejo 8). Bilbao.

\section{GUTIÉRREZ, F.I.}

2009 La explotación de moluscos y otros recursos litorales en la Región Cantábrica durante el Pleistoceno final y el Holoceno inicial. Publican, Universidad de Cantabria. Santander.

\section{IBAÑEZ, M}

1982 Sistemática y ecología del género Patella en la costa vasca: I. Lurrarde, 5, 69-78.

IBAÑEZ, M., FELIÚ, J.

1983 Sistemática y ecología del género Patella en la costa vasca: II. Lurrarde, 6, 191-202.

IRIARTE, M.J., ARRIZABALAGA, A., ETXEBERRIA, F. y HERRASTI, L.

2005 La inhumación humana en conchero de J3 (Hondarribia, Gipuzkoa). En: ARIAS, P., et al. (Eds.) III Congreso del Neolítico en la Península Ibérica: 607-613. Universidad de Cantabria (Monografías del IIIPC, 1), Santander.

IRIARTE, M.J., ARRIZABALAGA, A., ETXEBERRIA, F., HERRASTI, L. y ÁLVAREZ-FERNÁNDEZ, E.

2010 Shell Midden people in Northern Iberia. New data from the Mesolithic rock shelter of J3 (Basque Country, Spain). Zephyrus, LXV(1), 117-127

\section{MADARIAGA, B.}

1975 Estudio de la fauna marina de la Cueva de Tito Bustillo (Oviedo). En: MOURE, J. A. (ed.) Excavaciones en la Cueva de "Tito Bustillo" (Ribadesella, Asturias): Campañas de 1972 y 1974: 89-108. Instituto de Estudios Asturianos. Oviedo.

1976 Los fenómenos de productividad alimentaria en el Neolítico. Anales del Instituto de Estudios Agropecuarios, II: 176-212. 
MADINA, M. (Ed.)

2010 Protección de la zona marina frente a Jaizkibel y Ulía, Euskadi. Propuesta científica para la creación de un corridor ecológico marino entre Donostia (Ulía) y Biarritz, mediante su inclusion en la Red Natura 2000. Oceana.

MARTÍNEZ, J. y ADARRAGA, I.

2006 Programa de vigilancia y control de la introducción de especies invasoras en ecosistemas litorales de la Costa Vasca. 1. Costa de Gipuzkoa. Sociedad Cultural de Investigación Submarina. http://www.osakidetza.euskadi.net/ r8520319/eu/contenidos/informe_estudio/invasoras_costa/ eu_doc/adjuntos/memoria.pdf

\section{MORENO, R}

1994 Análisis arqueomalacológicos en la Península Ibérica. Contribución metodológica y biocultural. Tesis Doctoral. Departamento de Biología. Universidad Autónoma de Madrid. Madrid. (inédita).

\section{MORTENSEN, T.}

1927 Handbook of the Echinoderms of the British Isles. OUP, Oxford.
RELINI, G.

1980 Cirripedi toracici. Guide per il riconoscimento delle specie animali delle acque lagunari e costiere italiane. Consiglio Nazionale delle Ricerche. Rome.

SOUTHWARD, A.J.

2008 Barnacles. Published for The Linnean Society of London and The Estuarine and Coastal Sciences Association by Field Studies Council (Synopses of the British Fauna, New Series 57). London.

SOUTHWARD, E.C. y CAMPBELL, A.C.

2005 Echinoderms. Published for The Linnean Society of London and The Estuarine and Coastal Sciences Association by Field Studies Council (Synopses of the British Fauna, New Series 56). London. 